\title{
Comparative Study of Recurrence and Complications Using Various Sclerosants by Single Dart Technique in Treatment of Ganglion Cysts
}

\author{
Shirol S S ${ }^{2} \cdot$ Harini B $S^{1} \cdot$ Nagarajrao Sanu ${ }^{2} \cdot$ Mohammed Mansoor Ahmed ${ }^{1}$
}

Received: 25 February 2016 / Accepted: 22 April 2016/Published online: 21 May 2016

(C) Association of Surgeons of India 2016

Sir,

We read with a lot of interest the article published in your esteemed journal titled "Comparative study of recurrence and complications using various sclerosants by single dart technique in treatment of ganglion cysts" [1]. We would like to congratulate the authors for the excellent results with minimal recurrence rate. Ganglions are very common soft tissue swelling of the wrist and hand, which could be asymptomatic or present with aesthetic concerns or pain. The treatment described varies from reassurance, aspiration, intra-lesional sclerosants, steroids, hyaluronidase, threading, and surgical excision. However, the ganglions are notoriously known for recurrence. The recurrence rate reported varies with each published literature and with each modality ranging from 0 to $94 \%$ [2]. Becker suggested the use of steroid injection in treating ganglion, with an $87 \%$ resolution rate, based on the initial theory that chronic inflammatory may take part in the pathogenesis of ganglion; however, the subsequently published literature was with variable results [3]. Here, the title of the article published mentions it as recurrence and complications with various sclerosants, which is contrary to the action of steroids; the steroids act as anti-inflammatory agents, whereas sclerosants act by inducing inflammation and fibrosis. Hyaluronidase is a naturally occurring enzyme which depolymerizes hyaluronic acid, a viscous substance abundantly present in a mucinous ganglion with

Shirol S S

ssshirol@yahoo.co.uk

1 Department of General Surgery, Karnataka Institute of Medical Sciences, Hubli, India

2 Sampige Superspecialty Clinic, Opp. Vasan Eye Care Hospital, Club Road, Near Court Circle, Hubli, Karnataka 580028, India variable recurrence reported; again, a point of note is that it is not a sclerosant.

Since both triamcinolone and hyaluronidase do not belong to a group of sclerotherapeutic agents, the article title seems to be inappropriate and misleading for the readers. Hence, we suggest that the article title should be interpreted as "Comparative study of recurrence and complications using various agents by single dart technique in treatment of ganglion cysts" which is more scientific.

\section{Compliance with Ethical Standards}

Conflict of Interest The authors declare that they have no conflict of interest.

Funding None

\section{References}

1. Chatterjee S, Basu A, Gupta S, Biswas S (2014) Comparative study of recurrence and complications using various sclerosants by single dart technique in treatment of ganglion cysts. Indian J Surg 76(5): 350-353

2. Becker WF (1953) Hydrocortisone therapy in ganglia. Indusi Med 22:555-557

3. Suen M, Fung B, Lung CP (2013) Treatment of ganglion cysts. ISRN Orthop 28:2013 